\title{
American Put Option Pricing for Stochastic-Volatility, Jump-Diffusion Models*
}

\author{
Floyd B. Hanson ${ }^{\dagger}(2441)$ and Guoqing $\operatorname{Yan}^{\dagger}(52827)$
}

\begin{abstract}
The numerical treatment for the American put option pricing is discussed for a stochastic-volatility, jumpdiffusion (SVJD) model with log-uniform jump amplitudes. Heston's (1993) mean reverting, square-root stochastic volatility model is used along with our uniform jump-amplitude model. However, computation is needed for nonlinear and multidimensional terms with dependence on the combined stock and volatility state space. A systematic finite difference formulation of the American put partial integro-differential complementary problem (PIDCP) is implemented using a successive overrelaxtion method projected on the maximum payoff function. Interpolation is used to construct the smooth transition to the payoff of the corresponding free boundary problem. Also, a fast, but less accurate, heuristic quadratic approximation, originally due to MacMillan (1986), is corrected and extended from pure diffusion models. The fast and simple quadratic approximation is compared with a more accurate PIDCP formulation. The simple quadratic approximation is briefly compared with market data.
\end{abstract}

Key words: stochastic volatility, jump diffusion, uniform jump-amplitudes, American option put pricing, quadratic approximation, linear complementary problem.

\section{INTRODUCTION}

The objective of this paper is to derive a quadratic approximation for computing the valuation for American options when the price of the underlying asset evolves as a stochastic volatility and jump diffusion (SVJD) model with log-uniform jump amplitudes [21], [22]. It has been clear that the classical Black-Scholes-Merton model [7], [15] fails to reflect the empirical facts: market return data display excess kurtosis (peaked and fat tailed distributions), skewness, volatility clustering and large, sudden movements in prices. These observations reveal that a simple geometric Brownian motion process misses some important features of the data. As discussed in Andersen, Benzoni and Lund [1], Bates [6] and Bakshi, Cao and Chen [3], the most reasonable model of stock prices would include both stochastic-volatility and jump-diffusion.

Most options traded on market are American-style [5], so being able to price these contingent claims is clearly

*This work is supported in part by the National Science Foundation under Grants DMS-02-07081 and DMS-02-02815, the latter was reseach support for G.Yan from C. Knessl. Any conclusions or recommendations expressed in this material are those of the authors and do not necessarily reflect the views of the National Science Foundation. Proceedings of 2007 American Control Conference, NYC, to appear 11 July in invited session WeA12.1 Stochastic Theory and Control in Finance, Part I, dedicated to Ioannis Karatzas.

$\dagger$ Department of Mathematics, Statistics, and Computer Science University of Illinois at Chicago, $851 \mathrm{~S}$ Morgan St.; m/c 249, Chicago, IL 60607-7045, USA; E-Mail: hanson@math.uic.edu or gyan2@math.uic.edu of practical importance. American-style options have the additional feature that exercise is permitted at any time during the life of the option. This feature makes it more difficult to analyze than the corresponding European-style options. Unlike the European-style option pricing problem, formal-closed form solutions for the American option pricing problem have not been found even for pure diffusion models [12], [22]. The pricing of American options has usually resorted to finite-difference, binomial, or compoundoption approximation methods. These numerical methods are usually cumbersome and computationally costly to use. Since the price of an American call option on a nondividend paying security coincides with the corresponding European call option price for the jump-diffusion model, we will concentrate on the case of American put options here. However, see the forthcoming paper of Chiarella and Ziogas [8] for stochastic volatility models.

\section{Stochastic-Volatility Jump-Diffusion Model}

In contrast to the complete market setting of Black-Scholes as established by Harrison and Pliska [11] in the continuoustime case, the additional sources of uncertainty: the random jump sizes and stochastic volatility, introduced in our underlying price dynamics make the market incomplete with respect to the risk-free bank account, the underlying asset, and the finite number of options contracts. Consequently, the risk neutral probability measure alternative is not unique.

In this incomplete market setting, the underlying security price $S(t)$, the risky asset, is assumed under a candidate "risk-neutral" measure for risk-free interest rate $r$ :

$$
\begin{aligned}
d S(t)=S(t) & \left((r-\lambda \bar{J}) d t+\sqrt{V(t)} d W_{s}(t)\right. \\
+ & \left.\sum_{j=1}^{d N(t)} J\left(Q_{j}\right)\right),
\end{aligned}
$$

with Heston's [12], [6] mean-reverting stochastic volatility with parameter set $\left(\kappa_{v}, \theta, \sigma_{v}\right)$ :

$$
d V(t)=\kappa_{v}(\theta-V(t)) d t+\sigma_{v} \sqrt{V(t)} d W_{v}(t),
$$

where $W_{s}(t)$ and $W_{v}(t)$ are standard (i.e., zero-mean and unit-variance) Brownian motions for $S(t)$ and $V(t)$, respectively, such that the correlation is

$$
\operatorname{Corr}\left[d W_{s}(t), d W_{v}(t)\right]=\rho .
$$

The $N(t)$ is a Poisson process with common mean and variance,

$$
\mathrm{E}[d N(t)]=\lambda d t=\operatorname{Var}[d N(t)],
$$

intensity $\lambda$, jump-amplitude $J(Q)$ and, given the $j$ th Poisson jump, $Q=Q_{j}$ is the $j$ th underlying mark random variable 
$Q_{j}$ which is taken from a set of independent, identically distributed (IID) random variables. The form

$$
Q=\ln (J(Q)+1)
$$

is the underlying mark chosen to simplify the log-return $\ln (S(t))$ representation. For an accessible general formulation of applied stochastic processes with jump-diffusions see Hanson [10].

It is only necessary to know that the risk-neutral measure exists [13]. Since Scott [17] finds that interest rate volatility has little impact on short-term option prices, the interest rate $r$ will be assumed constant in this paper.

Let the density of the jump-amplitude be log-uniformly distributed in the mark variable $Q$ on $[a, b]$ :

$$
\phi_{Q}(q)=\frac{1}{b-a}\left\{\begin{array}{ll}
1, & a \leq q \leq b \\
0, & \text { else }
\end{array}\right\},
$$

where $a<0<b$. The mark $Q$ has moments, such that the mean is

$$
\mu_{Q} \equiv \mathrm{E}_{Q}[Q]=0.5(b+a)
$$

and variance is

$$
\sigma_{Q}^{2} \equiv \operatorname{Var}_{Q}[Q]=(b-a)^{2} / 12 .
$$

Thus, the original jump-amplitude $J$ has mean

$$
\bar{J} \equiv \mathrm{E}[J(Q)]=(\exp (b)-\exp (a)) /(b-a)-1,
$$

which is used in the risk-neutral analysis later.

There are several reasons for choosing the log-uniform distribution: 1) since the exponentially small tails of the lognormal or log-double-exponential distribution are contrary to the flat and thick tails of the long time financial market logreturn data; 2) around the near-zero peak of the log-doubleexponential and the log-normal, the jumps are small, so are not qualitatively different or separately detectable from the continuous diffusion fluctuations; 3 ) an infinite jump domain is unrealistic, since the jumps should be bounded in real world financial markets and, in fact, trading circuit breakers have been in place at the New York Stock Exchange [2] since 1988 the shut down trading in several stages during extreme events to avoid crashes like that in 1987 [5]; 4) an infinite jump domain leads to unrealistic restrictions in portfolio optimization and, in particular, Zhu and Hanson [23] show that for an infinite domain jump-amplitude distribution the instantaneous stock fraction is constrained on $[0,1]$ while borrowing and short-selling restriction can vary widely in the case of sufficiently small crash $|a|$ and rally $b$ limits.

The square-root stochastic-volatility process, Eq. (2), has two major advantages. First, the model can allow for systematic volatility risk. The second is that the process generates an analytically tractable method of pricing options without sacrificing accuracy of requiring undesirable restrictions on parameter values according to Bates [6].

By the Itô's chain rule and under a risk-neutral probability measure $\mathcal{M}$, the log-return process $\ln (S(t))$ satisfies the SDE

$$
\begin{gathered}
d \ln (S(t))=(r-\lambda \bar{J}-V(t) / 2) d t+\sqrt{V(t)} d W_{s}(t) \\
+\sum_{j=1}^{d N(t)} Q_{j} .
\end{gathered}
$$

\section{AMERICAN Put Option Price}

Compared with European-style options, American-style options have the additional feature that exercise is permitted at any time during the life of the option, $[0, T]$, with strike price $K$. Since the price of an American call option on a non-dividend paying security in the case of a jump-diffusion model coincides with the corresponding European call option's price, which has a closed analytic form solution in terms of a characteristic function or Fourier transform [22], we will concentrate on the case of American put options here. The determination of the early exercise of American put options is gives rise to a free boundary problem.

The price of the American put option will have the same description as a European put option in the regular region and it will be equal to the intrinsic value in the optimal exercise region. The American put option price can be specified as

$$
\begin{aligned}
P^{(A)}(S(t), V(t), t ; K, T)= & \sup _{\tau \in \mathcal{T}(t, T)}\left[\mathrm { E } \left[e^{-r(\tau-t)}\right.\right. \\
& \left.\left.\cdot \max [K-S(\tau), 0] \mid \mathcal{F}_{t}\right]\right]
\end{aligned}
$$

on the domain $\mathcal{D}=\{(s, t) \mid[0, \infty) \times[0, T]\}$, where $K$ is the strike price, $T$ is the maturity date, $\mathcal{T}(t, T)$ are a set of stopping times $\tau$ satisfying $t<\tau \leq T$.

Typically at fixed time $t$ there is a value of $S$ which marks the boundary between two regions: regular region and optimal exercise region. We denote this free boundary by the critical curve $S^{*}=S^{*}(t)$ for $t \in[0, T]$. Hence, the free boundary $s=S^{*}(t)$ in the $(s, t)$-plane separates the domain $\mathcal{D}$ into two regions: the continuation region $\mathcal{C}$, where it is optimal to hold the option, i.e.,

$$
\text { if } s>S^{*}(t) \text {, then } P^{(A)}(s, v, t ; K, T)>\max [K-s, 0] \text {, }
$$

so $P^{(A)}$ will have the same discription as the European price $P^{(E)}$ at least in the jump-diffusion case and the exercise region $\mathcal{E}$, where it is optimal to exercise the option, i.e.,

$$
\text { if } s \leq S^{*}(t) \text {, then } P^{(A)}(s, v, t ; K, T)=\max [K-s, 0] \text {. }
$$

The partial integro-differential equation (PIDE) that American put option satisfies is similar to that of the European option (Yan and Hanson [22]), letting $s=S(t)$ and $v=$ $V(t)$, in regular region (prior to optimal, early exercise) is:

$$
\begin{aligned}
0= & \frac{\partial P^{(A)}}{\partial t}(s, v, t ; K, T)+\mathcal{A}\left[P^{(A)}\right](s, v, t ; K, T) \\
\equiv & \frac{\partial P^{(A)}}{\partial t}+(r-\lambda \bar{J}) s \frac{\partial P^{(A)}}{\partial s}+k_{v}\left(\theta_{v}-v\right) \frac{\partial P^{(A)}}{\partial v} \\
& +\frac{1}{2} v s^{2} \frac{\partial^{2} P^{(A)}}{\partial s^{2}}+\rho \sigma_{v} v s \frac{\partial^{2} P^{(A)}}{\partial s \partial v}+\frac{1}{2} \sigma_{v}^{2} v \frac{\partial^{2} P^{(A)}}{\partial v^{2}} \\
& -r P^{(A)}+\lambda \int_{-\infty}^{\infty}\left(P^{(A)}\left(s e^{q}, v, t ; K, T\right)\right. \\
& \left.-P^{(A)}(s, v, t ; K, T)\right) \phi_{Q}(q) d q,
\end{aligned}
$$

for $(s, t) \in \mathcal{C}$ and defining the backward operator $\mathcal{A}$.

The American put option pricing problem as a free boundary problem is given by

$$
0=\frac{\partial P^{(A)}}{\partial t}(s, v, t ; K, T)+\mathcal{A}\left[P^{(A)}\right](s, v, t ; K, T)
$$


for $(s, t) \in \mathcal{C} \equiv\left[S^{*}(t), \infty\right) \times[0, T]$

$$
0>\frac{\partial P^{(A)}}{\partial t}(s, v, t ; K, T)+\mathcal{A}\left[P^{(A)}\right](s, v, t ; K, T)
$$

for $(s, t) \in \mathcal{E} \equiv\left[0, S^{*}(t)\right] \times[0, T]$. where critical stock price $S^{*}(t)$ is not known a priori as a function of time.

The fixed and smooth free boundary conditions are

$$
\begin{gathered}
\lim _{t \rightarrow T} P^{(A)}(s, v, t ; K, T)=\max (K-s, 0), \\
\lim _{s \rightarrow 0} P^{(A)}(s, v, t ; K, T)=K, \\
\lim _{s \rightarrow \infty} P^{(A)}(s, v, t ; K, T)=0, \\
\lim _{s \rightarrow S^{*}(t)} P^{(A)}(s, v, t ; K, T)=K-S^{*}(t), \\
\lim _{s \rightarrow S^{*}(t)} \partial P^{(A)}(s, v, t ; K, T) / \partial s=-1 .
\end{gathered}
$$

\section{Finite Differences for AmericAn Put Option LINEAR COMPLEMENTARITY PROBLEM}

Other methods for the American option are based on the linear complementarity problem (LCP) formulation [9], [18], [19], [20] and will be used to check the accuracy of the quadratic approximation formula. The finite difference version of the LCP formulation finds the maximum of the successive overrelaxation (SOR) approximation, and hence is called the projected successive overrelaxation (PSOR is SOR projected on to the maximum) approximation. The payoff at each discrete point $\left(S_{i}, V_{j}, t_{k}\right)$ using

$$
\max \left(S O R\left[P^{(A)}\right]_{i, j, k}, K-S_{i}\right),
$$

with corresponding constraints. The optimal exercise boundary $S^{*}$ is automatically captured by this information and can be determined afterward. Solutions by the PSOR version of LCP can be obtained for the full SVJD problem without using the quadratic or quasi-deterministic volatility approximations.

When the free boundary problem is transferred to partial integro-differential complementarity problem (PIDCP) it is formulated as follows

$$
\begin{gathered}
P^{(A)}(s, v, t ; K, T)-F(s) \geq 0, \\
\frac{\partial P^{(A)}}{\partial \tau}-\mathcal{A} P^{(A)} \geq 0, \\
\left(\frac{\partial P^{(A)}}{\partial \tau}-\mathcal{A} P^{(A)}\right)\left(P^{(A)}-F\right)=0,
\end{gathered}
$$

where $F(s)=\max [K-s, 0]$ is the put objective and $\tau=$ $T-t$ is the time-to-go. In addition there are the contact and tangent free boundary conditions in the last two equations of (8).

For the discretization, the Crank-Nicolson implicit method is used with

$$
\begin{gathered}
P^{(A)}\left(S_{i}, V_{j}, T-\tau_{k} ; K, T\right)=U\left(S_{i}, V_{j}, \tau_{k}\right) \simeq U_{i, j}^{(k)}, \\
U^{(k)}=\left[U_{i, j}^{(k)}\right],
\end{gathered}
$$

time-step $\Delta \tau$, the discretization of the operator $\mathcal{A} \simeq L$,

$$
\frac{\partial P^{(A)}}{\partial \tau} \simeq \frac{\left(U^{(k+1)}-U^{(k)}\right)_{i, j}}{\Delta \tau}
$$

and

$$
\mathcal{A} P^{(A)} \simeq \frac{1}{2} L\left(U^{(k+1)}+U^{(k)}\right) .
$$

Using standard linear algebraic definitions, letting

$$
\widehat{\mathbf{U}}^{(k)}=\left[\widehat{U}_{i}^{(k)}\right]
$$

the single subscripted version of $U^{(k)}=\left[U_{i, j}^{(k)}\right]$, with corresponding $\widehat{\mathbf{F}}, \widehat{L}, \widehat{M}$ and $\widehat{\mathbf{b}}^{(k)}$, so

$$
\widehat{M} \equiv I-\frac{\Delta \tau}{2} \widehat{L}
$$

and

$$
\widehat{\mathbf{b}}^{(k)} \equiv\left(I+\frac{\Delta \tau}{2} \widehat{L}\right) \widehat{\mathbf{U}}^{(k)},
$$

leads to a discretized, linear algebraic complementarity problem (see Wilmott et al. [18], [19], Wilmott [20] or Cottle et al. [9] for more general LCPs):

$$
\begin{gathered}
\widehat{\mathbf{U}}^{(k+1)}-\widehat{\mathbf{F}} \geq \mathbf{0}, \quad \widehat{M} \widehat{\mathbf{U}}^{(k+1)}-\widehat{\mathbf{b}}^{(k)} \geq \mathbf{0}, \\
\left(\widehat{\mathbf{U}}^{(k+1)}-\widehat{\mathbf{F}}\right)^{\top}\left(\widehat{M} \widehat{\mathbf{U}}^{(k+1)}-\widehat{\mathbf{b}}^{(k)}\right)=0,
\end{gathered}
$$

The projective successive overrelaxation (PSOR) algorithm with acceleration parameter $\omega$ for LCP (10) t by iterating $\widetilde{U}_{i}^{(n+1)}$ for $\widehat{U}_{i}^{(k+1)}$ until changes are sufficiently small:

$$
\begin{aligned}
\tilde{U}_{i}^{(n+1)} & =\max \left(\widehat{F}_{i}, \widetilde{U}_{i}^{(n)}+\omega \widehat{M}_{i, i}^{-1}\left(\widehat{b}_{i}^{(k)}\right.\right. \\
& \left.\left.-\sum_{j<i} \widehat{M}_{i, j} \widetilde{U}_{j}^{(n+1)}-\sum_{j \geq i} \widehat{M}_{i, j} \widetilde{U}_{j}^{(n)}\right)\right) .
\end{aligned}
$$

For the discretization of the PIDE, the first-order and secondorder spatial derivatives and the cross-derivative term are all approximated with the standard second-order accurate finite differences, using a nine-point computational molecule. Linear interpolation is applied to the jump integral term.

Quadratic extrapolation of the solution is used for the smooth contact condition in critical stock price $S^{*}(t)$ calculation. Suppressing the dependence on $(v, \tau)$ in the solution, let $u_{i}=U\left(S_{i}, V_{j}, \tau_{k}\right)$ and then selecting the index $i$ such that $u_{i}=F_{i}=F\left(S_{i}\right)$, the exercise payoff, and $u_{i+\ell}>F_{i+\ell}$ for $\ell=1: 2$, so are two nearby values in the continuation region. Choosing the quadratic extrapolation function to be

$$
U_{q}(S)=c_{0}+c_{1}\left(S-S_{i+1}\right)+0.5 c_{2}\left(S-S_{i+1}\right)^{2}
$$

satisfying the interpolation conditions $U_{q}\left(S_{i+\ell}\right)=u_{i+\ell}$ for $\ell=1: 2$ and the smooth contact conditions

$$
U_{q}\left(S^{*}\right)=F\left(S^{*}\right)
$$

with

$$
U_{q}^{\prime}\left(S^{*}\right)=-1,
$$

then the critical stock price satisfies,

$$
S^{*} \simeq S_{i+1}-\frac{d_{2}}{d_{1}}\left(1+\sqrt{1+\frac{d_{2}}{d_{1}} d S}\right),
$$


where $d_{1}=\left(U_{i+2}-U_{i+1}\right) / d S$ and $d_{2}=U_{i+1}-F_{i+1}$. Note that the extrapolation problem has four unknowns, $\left\{c_{0}, c_{1}, c_{2}, S^{*}\right\}$, to match the four given conditions, assuming that the contact is within $\left(S_{i}, S_{i+1}\right)$.

Sample output for the American put option price approximated by the LCP method is given in Figure 1 versus moneyness, $S / K$, for $\tau=T-t=0.5,0.25,0.10$ and 0 years before maturity. Note the smooth-appearing transition to the payoff function when $\tau>0$. The corresponding results for the critical stock price at early exercise is given in Fig. 2 versus the time before maturity in years, parameterized by various value of the stochastic variance, $V=0.04,0.1,0.2$, 0.4 and 0.8 .

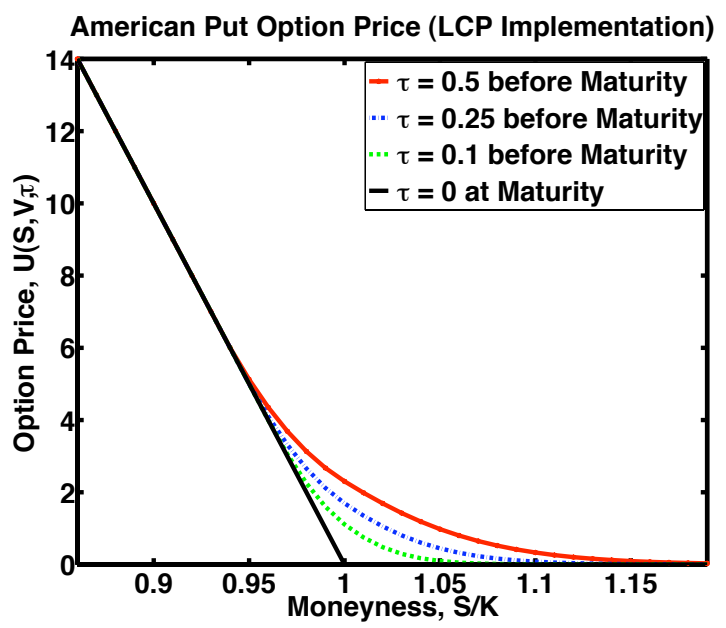

Fig. 1. PSOR finite difference implementation of LCP gives SVJD-Uniform American put option prices $U(S, V, \tau)=P_{L C P}^{(A)}$.

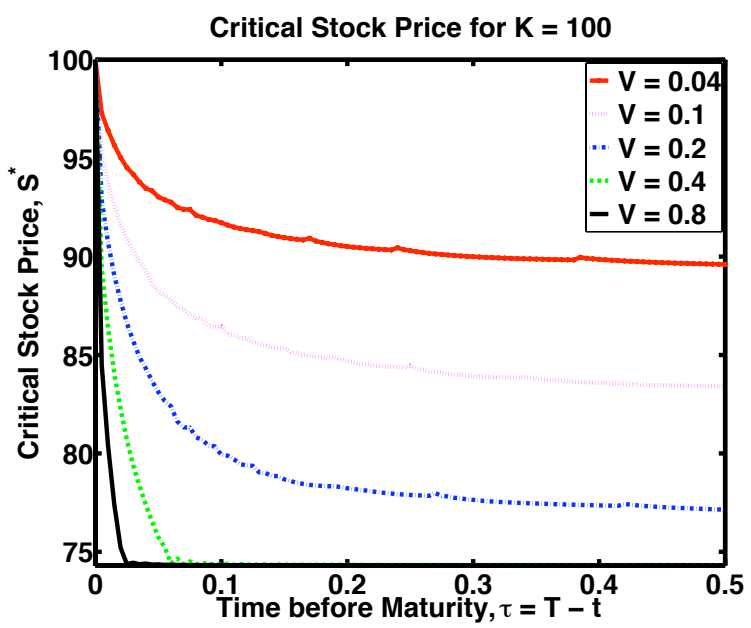

Fig. 2. PSOR finite difference critical stock prices $S^{*}(\tau ; V)$, (using quadratic extrapolation approximations wth smooth contact to the payoff function).

\section{Heuristic Quadratic APPROXIMATION FOR AMERICAN PUT OPTION}

A simple and fast heuristic quadratic approximation is used to derive an implicit analytic solution, which was originally proposed by MacMillan [14], and then corrected and extended by Barone-Adesi and Whaley [4] and Bates [5]. Here, we extend it to the stochastic-volatility, jump-diffusion model with log-uniformly distributed jump-amplitudes. The key insight into the quadratic approximation approach is that, if the PIDE applies to American options as well as European options $P^{(E)}$, it also applies to the optimal exercise premium of the American option over the European option. For an American put option and the quadratic approximation, the early exercise premium $\varepsilon^{(P)}$ is defined using a common current space-time $(s, t)$ and common strike price $K$ as

$$
\begin{aligned}
\varepsilon^{(P)}(s, v, t ; K, T) \equiv & P^{(A)}(s, v, t ; K, T) \\
& -P^{(E)}(s, v, t ; K, T),
\end{aligned}
$$

where $P^{(E)}$ is given by Fourier inverse in Yan and Hanson [22]. Without losing much generality, the early premium can be written in MacMillan's [14] ad hoc time-dependent form with function $G(t)$ as

$$
\varepsilon^{(P)}(s, v, t ; K, T) \simeq G(t) Y(s, v, G(t)) .
$$

Choosing the transformed time

$$
G(t) \equiv 1-\exp (-r(T-t))
$$

so it is zero when $t=T$, also making the premium value of $\varepsilon^{(P)}(s, v, t ; K, T)$ zero. The approximation of the early exercise premium PIDE is, after dropping the $G$-derivative term, since the quadratic coefficient is bounded,

$$
0 \leq-G^{\prime} G / r=(1-G) G \leq 0.25,
$$

making $G(t)$ a parameter rather than a variable,

$$
\begin{aligned}
0= & +(r-\lambda \bar{J}) s \frac{\partial Y}{\partial s}-\frac{r}{G} Y+\kappa_{v}(\theta-v) \frac{\partial Y}{\partial v} \\
& +\frac{1}{2} v s^{2} \frac{\partial^{2} Y}{\partial s^{2}}+\rho \sigma_{v} v s \frac{\partial^{2} Y}{\partial s \partial v}+\frac{1}{2} \sigma_{v}^{2} v \frac{\partial^{2} Y}{\partial v^{2}} \\
& +\lambda \int_{-\infty}^{\infty}\left(Y\left(s e^{q}, v, G\right)-Y(s, v, G)\right) \phi_{Q}(q) d q
\end{aligned}
$$

with the corresponding large asset and free boundary conditions for $Y(s, v, G)$ for fixed $t$ or $G$ :

$$
\lim _{s \rightarrow \infty} Y(s, v, G(t))=0,
$$

$$
\begin{aligned}
& \lim _{s \rightarrow S^{*}} Y(s, v, G)=\frac{1}{G}\left(K-S^{*}-P^{(E)}\left(S^{*}, v, t\right)\right), \\
& \lim _{s \rightarrow S^{*}} \frac{\partial Y(s, v, G)}{\partial s}=\frac{1}{G}\left(-1-\frac{\partial P^{(E)}}{\partial s}\left(S^{*}, v, t\right)\right) .
\end{aligned}
$$

Reformulating Bates' [6] constant-volatility jumpdiffusion (CVJD) ad hoc approach, the dependence on the volatility variable $v$ is assumed to be weak and is replaced by the quasi-deterministic-volatility jump-diffusion (QDVJD) approximation of $V(t)$, such that

$$
d \bar{V}(t)=\mathrm{E}[d V(t) \mid V(t)=\bar{V}(t)]=\kappa_{v}(\theta-V(t)) d t
$$

and

$$
\bar{V}(t) \equiv \theta+(V(0)-\theta) e^{-\kappa_{v} t},
$$


so that the $v$-derivatives disappear letting

$$
v=\overline{\bar{V}}=\int_{0}^{T} \bar{V}(t) d t / T
$$

and

$$
Y(s, \overline{\bar{V}}, G) \simeq \widehat{Y}(s)
$$

satisfies the linear equi-dimensional OIDE ( $G$ and $\bar{V}$ are suppressed parameters),

$$
\begin{aligned}
0= & (r-\lambda \bar{J}) s \widehat{Y}^{\prime}(s)-r \widehat{Y}(s) / G+\overline{\bar{V}} s^{2} \widehat{Y}^{\prime \prime}(s) / 2 \\
& +\lambda \int_{-\infty}^{\infty}\left(\widehat{Y}\left(s e^{q}\right)-\widehat{Y}(s)\right) \phi_{Q}(q) d q .
\end{aligned}
$$

The solution has the power form

$$
\widehat{Y}(s)=c_{1} s^{A_{1}}+c_{2} s^{A_{2}},
$$

where $A_{1}$ and $A_{2}$ are solutions to

$$
\begin{aligned}
0= & \overline{\bar{V}} A^{2} / 2+(r-\lambda \bar{J}-\overline{\bar{V}} / 2) A-r / G \\
& +\lambda\left(\left(e^{b A}-e^{a A}\right) /((b-a) A)-1\right),
\end{aligned}
$$

assuming the log-uniform jump-distribution (3). Then combining with boundary conditions, taking only negative root $A_{2}$, say, so that $S^{*}$ is the solution to the implicit equations for each fixed $t \leq T_{e}$ :

$$
\begin{aligned}
K-S^{*}- & P^{(E)}\left(S^{*}, \overline{\bar{V}}, t\right)= \\
& \frac{S^{*}}{A_{2}}\left(-1-\left(\frac{\partial P^{(E)}}{\partial s}\right)\left(S^{*}, \overline{\bar{V}}, t\right)\right) .
\end{aligned}
$$

Also, $c_{2}$ is given by

$$
c_{2}=\left(K-S^{*}-P^{(E)}\left(S^{*}, \overline{\bar{V}}, t\right)\right) /\left(G \cdot\left(S^{*}\right)^{A_{2}}\right) .
$$

This completes the crucial steps of the approximate quadratic approximation with QDVJD volatility approximation.

Sample results for the quadratic approximation (QA) of both American and European put option prices are given in Fig. 3 for $T=0.5$ years with respect to the moneyness, $S / K$. The difference between the two curves is the quadratic approximation to the early exercise premium $\varepsilon^{(P)}$.

\section{COMPARISONS AND CALIBRATIONS}

The heuristic quadratic approximation (QA) for the American put option price in an SVJD environment is briefly compared to that of the more accurate linear complementary (LCP) finite difference approximation (FD) in Fig. 4 with respect to the strike price $K$ when the time horizon is $T=0.1,0.25$ and 0.5 years. The maximum price difference $P_{Q A}^{(A)}-P_{L C P}^{(A)}$ is $\$ 0.08, \$ 0.14, \$ 0.21$ for $T=0.1,0.25$ and 0.5 years, respectively, so QA is probably good for practical purposes when a quick estimate is needed.

The quadratic approximation is compared against the options market in Fig. 5 using concurrent CBOE quotes on XEO for European put options and OEX for American put options from April 10, 2006. They are both based upon the same S\&P 100 index (SPX). The quadratic approximation is used to compute the approximation to the American put options prices corresponding to the OEX, while the

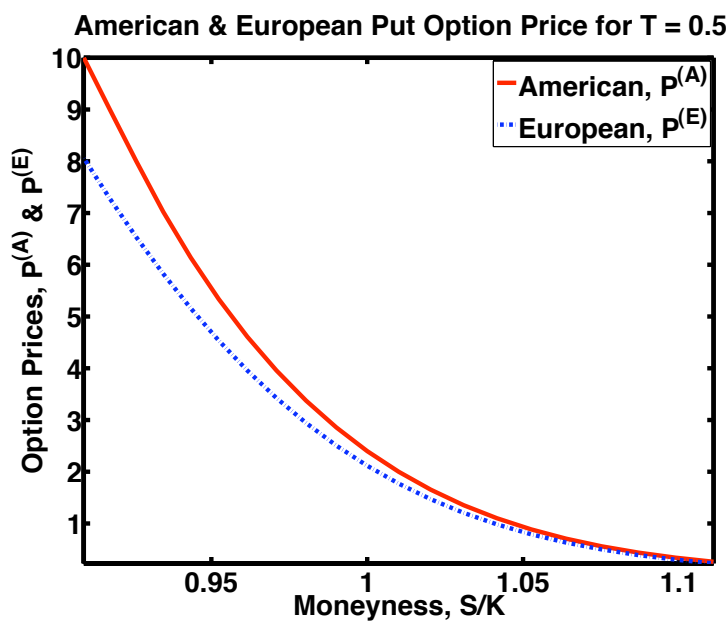

Fig. 3. The heuristic quadratic approximation gives SVJD-Uniform American $P^{(A)}=P_{Q A}^{(A)}$ compared European $P^{(E)}$ put option prices for $T=0.5$ years, with averaged approximation of $V(t)$.

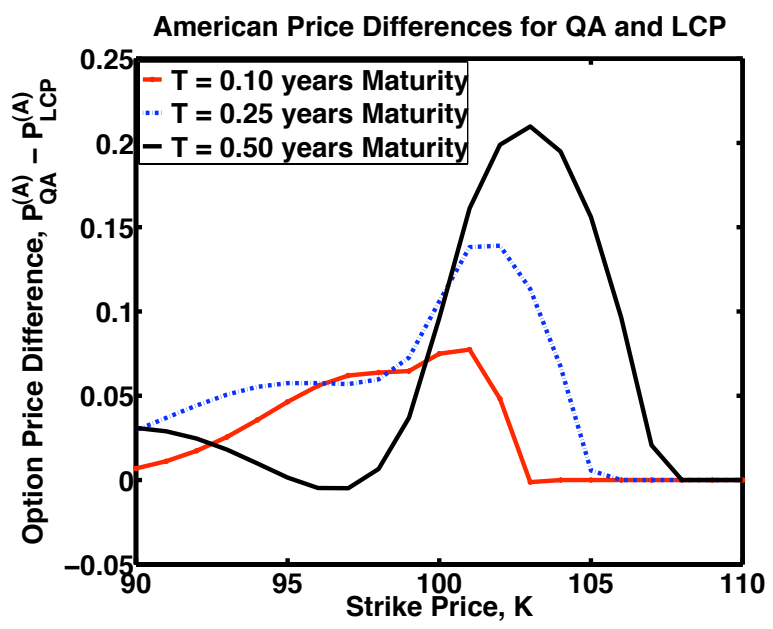

Fig. 4. Comparison of American put option prices evaluated by quadratic approximation (QA) and LCP finite difference (FD) methods when $S=$ $\$ 100$ and $V=0.01$ for $T=0.1,0.25$ and 0.5 years, respectively.

XEO data is used for the parameter calibration with the European option price analytical formula and hence in the early exercise premium. Then, the estimated parameters are used for calculating the American put option pricing [21]. A mean square error $\mathrm{MSE}=0.137$ was obtained using the calibrated parameters, so demonstrating a good fit. The maximum absolute price difference $P_{Q A}^{(A)}-P_{O E X}^{(A)}$ is $\$ 0.41$, $\$ 0.46, \$ 0.73, \$ 1.15, \$ 0.68$ for $T=11,39,67,102,168$ days, respectively. Sample SVJD and log-uniform jump parameters estimated from the XEO quotes [21] from April 10, 2006 are $k_{v}=10.62, \theta_{v}=0.0136, \sigma_{v}=0.175, \rho=-0.547, a=-$ $0.140, b=+0.011, \lambda=0.549$ and $v=0.0083$.

Since the parameters, including model structural parameters and option parameters like underlying price, strike price and maturity are very diversified, they are not suitable for calibration the LCP finite differences method against the CBOE market American option pricing data OEX [21]. The 


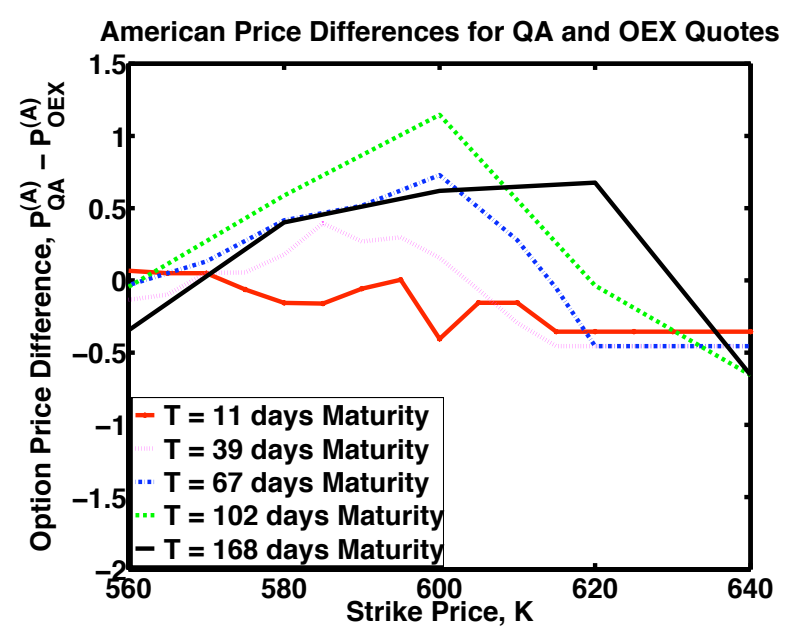

Fig. 5. Comparison of American put option prices evaluated by quadratic approximation (QA) method and OEX quotes when $S=\$ 100$ and $V=$ 0.01 for $T=11,39,67,102,168$ days, respectively. The OEX quotes are taken from the CBOE from April 10, 2006, the underlying S\&P 100 index (SPX) is 587.84.

difficulty is the numerical mismatch between the arbitrary parameter values and the finite difference lattice.

\section{SUMmary AND CONCLUSION}

An alternative stochastic-volatility jump-diffusion (SVJD) model is proposed with square root mean reverting for stochastic-volatility combined with log-uniform jump amplitudes.

The heuristic quadratic approximation (QA) and the LCP finite difference scheme for American put option pricing are compared, with QA being good for practical purposes.

The QA results are calibrated against real market American option pricing data OEX (with XEO for European price base) and yield reasonable results considering the simplicity of QA.

\section{REFERENCES}

[1] T.G. Andersen, L. Benzoni and J. Lund, "An Empirical Investigation of Continuous-Time Equity Return Models," Journal of Finance, vol. 57, 2002, pp. 1239-1284.

[2] Aourir, C. A., D. Okuyama, C. Lott and C. Eglinton. (2002). Exchanges - Circuit Breakers, Curbs, and Other Trading Restrictions, http://invest-faq.com/articles/ exch-circuit-brkr.html.

[3] G. Bakshi, C. Cao and Z. Chen, "Empirical Performance of Alternative Option Pricing Models," Journal of Finance,vol. 52, 1997, pp. 20032049.
[4] G. Barone-Adesi and R. E. Whaley, "Efficient analytic approximation of American Option Values," Journal of Finance, vol. 42, 1987, pp. 301-320.

[5] D. Bates,"The Crash of '87: Was It Expected? The Evidence from Option Markets," Journal of Finance, vol. 46, 1991, pp. 1009-1044.

[6] D. Bates, "Jump and Stochastic Volatility :Exchange Rate Processes Implict in Deutsche Mark in Options," Review of Financial Studies, vol. 9, 1996, pp. 69-107.

[7] F. Black and M. Scholes, "The Pricing of Options and Corporate Liabilities," J. Political Economy, vol. 81, 1973, pp. 637-659.

[8] C. Chiarella and A. Ziogas, "Pricing American Options under Stochastic Volatility," School of Finance and Economics, University of Technology, Sydney, AUS, forthcoming, 2006.

[9] R.W. Cottle, J.S. Pang, R.E. Stone, The Linear Complementarity Problem, Academic Press, New York, NY, 1992

[10] F. B. Hanson, Applied Stochastic Processes and Control for JumpDiffusions: Modeling, Analysis and Computation, SIAM Books, Philadelphia, PA, to appear 2007.

[11] Harrison, J. M., Pliska, S. R., "Martingales and stochastic integrals in the theory of continuous trading," Stochastic Processes and Their Applications, vol.11, 1981, pp. 215-260.

[12] S. L. Heston, A Closed-form Solution for Options with Stochastic Volatility with Applications to Bond and Currency Options, Review of Financial Studies, vol. 6, 1993, pp. 327-343.

[13] J. C. Hull, Options, Futures, \& Other Derivatives, 4th Edition, Prentice-Hall, Englewood Cliffs, NJ, 2000.

[14] L.W.MacMillan,"Analytic Approximation for the American Put Option," Advances in Futures and Options Research, vol. 1A, 1986, pp. 119-139.

[15] R. C. Merton, "Theory of Rational Option Pricing," Bell J. Econ. Mgmt. Sci., vol. 4, 1973, pp. 141-183.

[16] C. W. Oosterlee, On Multigrid for Linear Complementarity Problems with Application to American-Style Options, Electronic Transactions on Numerical Analysis, vol. 15, 2003, pp. 165-185.

[17] L. Scott,"Pricing Stock Options in a Jump-Diffusion Model with Stochastic Volatility and Interest Rates: Applications of Fourier Inversion Methods," Mathematical Finance, vol. 7 (4), 1997, pp. 413-424.

[18] P. Wilmott, J. Dewynne and S. Howison, Option Pricing: Mathematical Models and Computation, Oxford Financial Press, Oxford, UK, 1993.

[19] P. Wilmott, J. Dewynne and S. Howison, The Mathematics of Financial Derivatives: A Student Introduction, Cambridge University Press, Cambridge, UK, 1995

[20] P. Wilmott, Derivatives: The Theory and Practice of Financial Engineering, John Wiley, New York, 1998.

[21] G. Yan, "Option Pricing for a Stochastic-Volatility Jump-Diffusion Model," Ph.D. Thesis, Dept. Math., Stat., and Comp. Sci., Univ. Illinois at Chicago, 126 pages, 22 June 2006

[22] G. Yan and F. B. Hanson,"Option Pricing for a Stochastic-Volatility Jump-Diffusion Model with Log-Uniform Jump-Amplitudes,"Proc. American Control Conference, pp. 2989-2994, 14 June 2006

[23] Z. Zhu and F. B. Hanson, "Optimal Portfolio Application with Double-Uniform Jump Model," Stochastic Processes, Optimization, and Control Theory: Applications in Financial Engineering, Queueing Networks and Manufacturing Systems/A Volume in Honor of Suresh Sethi, International Series in Operations Research and Management Science, Vol. 94, H. Yan, G. Yin, Q. Zhang (Eds.), Springer Verlag, New York, NY, Invited chapter, 28 pages, June 2006. 